\title{
Avaliação do conhecimento da equipe de enfermagem no processo de esterilização de materiais cirúrgicos
}

\author{
Evaluation of the knowledge of the nursing team in the sterilization process of surgical \\ materials
}

\section{Evaluación del conocimiento del equipo de enfermería en el proceso de esterilización de materiales quirúrgicos}

Jenifer Maria da Silva Ribeiro ${ }^{1 *}$, Caroline Alves do Amaral ${ }^{1}$, Jessimone Pereira de Souza ${ }^{1}$, Sílvia Letícia Barbosa da Silva1, Rayana Gonçalves de Brito², Millena de Sousa Oliveira Negrão ${ }^{3}$, Joseni da Silva Pompeu ${ }^{4}$, Luana Taís de Jesus Santos Pedrosa ${ }^{1}$.

\section{RESUMO}

Objetivo: Avaliar o grau de conhecimento da equipe de enfermagem no processo de esterilização de materiais cirúrgicos. Métodos: Pesquisa de campo, exploratória e de abordagem quali-quantitativa. Os dados foram coletados através de questionário semi-estruturado aplicado aos 22 profissionais de enfermagem atuantes na Central de Material e Esterilização no mês de junho de 2018. Resultados: A oferta de treinamentos é considerada insuficiente, $45 \%$ dos entrevistados afirmaram realizá-la semestralmente, sendo que a qualificação individual (73\%) ocorre por meios externos. Acerca do processo de desinfecção e esterilização, $95 \%$ conceituaram corretamente, assim como dos artigos críticos e não críticos (64\%). Evidenciou-se também que apenas 14\% conheciam a finalidade do Teste Bowie e Dick quanto à eficácia do sistema de vácuo da autoclave. Conclusão: É necessário à implementação de capacitações periódicas, com ênfase no processamento dos materiais cirúrgicos e manuseio de inovações tecnológicas utilizadas para tornar o serviço mais hábil e seguro.

Palavras-chave: Avaliação, Educação continuada, Enfermagem, Infecção hospitalar.

\begin{abstract}
Objective: To evaluate the degree of knowledge of the nursing team in the sterilization process of surgical materials. Methods: Field research, exploratory and qualitative-quantitative approach. The data were collected through a semi-structured questionnaire applied to the 22 nurses working at central materials and sterilization in june 2018. Results: The offer of training is considered insufficient, $45 \%$ of respondents said they do it every six months, and the individual qualification (73\%) occurs by external means. Regarding the disinfection and sterilization process, $95 \%$ correctly conceptualized, as well as critical and non-critical articles (64\%). It was also found that only $14 \%$ knew the purpose of the Bowie and Dick Test for the effectiveness of the autoclave vacuum system. Conclusion: It is necessary to implement periodic training, with emphasis on the processing of surgical materials and handling of technological innovations used to make the service more skilled and safe.
\end{abstract}

Keywords: Evaluation, Continuing education, Nursing, Hospital infection.

${ }^{1}$ Centro Universitário da Amazônia (UNAMA). Santarém - Pará. *E-mail: jenifer_ribeiro06@hotmail.com ${ }^{2}$ Universidade Federal do Oeste do Pará (UFOPA). Faculdade Estácio do Amazonas. Manaus - Amazonas. ${ }^{3}$ Faculdade Brasil Amazônia (FIBRA), Escola Superior da Amazônia (ESMAZ). Belém - Pará.

${ }^{4}$ Faculdades Integradas do Tapajós (FIT). Santarém - Pará. 


\section{RESUMEN}

Objetivo: Evaluar el grado de conocimiento del equipo de enfermería en el proceso de esterilización de materiales quirúrgicos. Métodos: Investigación de campo, exploratoria y de abordaje cuali-cuantitativa. Los datos fueron recolectados a través de un cuestionario semiestructurado aplicado a los 22 profesionales de enfermería actuantes en la materiales centrales y esterilización en el mes de junio de 2018. Resultados: La oferta de capacitación se considera insuficiente, el $45 \%$ de los encuestados dijeron que lo hacen cada seis meses, y la calificación individual (73\%) se realiza por medios externos. En cuanto al proceso de desinfección y esterilización, el $95 \%$ se conceptualizó correctamente, así como los artículos críticos y no críticos (64\%). También se descubrió que solo el 14\% conocía el propósito de la Prueba Bowie y Dick para la efectividad del sistema de vacío en autoclave. Conclusión: Es necesario la implementación de capacitaciones periódicas, con énfasis en el procesamiento de los materiales quirúrgicos y el manejo de innovaciones tecnológicas utilizadas para hacer el servicio más hábil y seguro.

Palabras clave: Evaluación, Educación continuada, Enfermería, Infección hospitalaria.

\section{INTRODUÇÃO}

Segundo a Resolução da Diretoria Colegiada - RDC n 15 de 15 de março de 2012, a Central de Material de Esterilização (CME), "é uma Unidade funcional destinada ao processamento de produtos para saúde dos serviços de assistência médico-odonto-hospitalares" (BRASIL, 2012). Devido à responsabilidade funcional de distribuir materiais para todos os serviços de saúde, os setores tornam-se dependentes diretamente da CME (VASQUES JD e DAMASIO YLR, 2016). Logo, para que haja um funcionamento ideal, depende de um conjunto de medidas como estrutura física adequada, equipe capacitada, recursos de materiais e equipamentos próprios para a operacionalização em todas as etapas do processo (BORGHETI SP; VIEGAS K; CAREGNATO RCA, 2016).

Nesse sentido, a estrutura organizacional da CME é classificada em três formas: centralizada, semicentralizada e descentralizada (ARARUNA AB e POSSO MBS, 2014), sendo que as atividades desenvolvidas no setor envolvem o recebimento, limpeza de todos os artigos críticos, semicríticos e não crítico, secagem de maneira rigorosa, montagem das bandejas e pacotes com identificação do material, desinfecção através do álcool etílico a $70 \%$, glutaraldeído $2 \%$ ou o ácido peracético.

A esterilização é normalmente realizada através das autoclaves e deve ser diariamente monitorada através do teste Bowie e Dick cuja função é identificar as falhas no funcionamento da bomba de vácuo para atestar o sistema pré-vácuo avaliando assim as condições desse processo (TIPPLE AFV, et al., 2011; JACOBY AM, RECH K, ASCARI RA, 2016; ROCHA JMN, 2015).

É importante que a Central tenha uma posição de destaque dentro do hospital, pois é a partir da eficácia dos seus serviços que os riscos de infecções exógenas são minimizados, sendo que a permanência de microrganismos ao processo de esterilização pode ocorrer por falhas humanas, necessitando de investimento constante em qualificação, pesquisa e integração com as Unidades consumidoras responsáveis pelas tarefas de prevenção, diagnóstico e terapêutica aos clientes (FUSCO SFB e SPIRI WC, 2014).

Os trabalhadores de enfermagem, na casualidade, se deparam diante de um setor que requer domínio e especificidades, principalmente a compreensão do benefício da eficácia do seu trabalho, considerando assim, a complexidade e malefícios que um material esterilizado indevidamente pode causar para um usuário que já se apresenta enfermo (SILVA AC e AGUIAR BGCO, 2008).

No entanto, há um grande desafio para essa equipe de enfermagem no desempenho das atividades, pois, além da teoria confrontar o exercício da prática, a estrutura física e a desvalorização profissional por parte das Unidades consumidoras, impactam negativamente durante essa assistência indireta (ANJOS MAM e OLIVEIRA JC, 2016), acarretando danos à saúde física e psíquica como a baixa estima, absenteísmo e alta rotatividade na CME resultante da sobrecarga de trabalho (OURIQUES CM e MACHADO MÉ, 2013; BUGS TV, et al., 2017). 
Portanto, o profissional enfermeiro deve estar preparado para o gerenciamento dessa Unidade, através do conhecimento específico, qualificado e atualizado, atuando de forma dinâmica, com reconhecido perfil de líder de equipe e educador (HOYASHI CMT; RODRIGUES DCGA; OLIVEIRA MFA, 2015), com responsabilidade de supervisionar, planejar, elaborar instrumentos operacionais e administrar recursos materiais e humanos (GIL RF; CAMELO SH; LAUS AM, 2013). A importância da pesquisa se faz pela criticidade e especificidade da CME, bem como, a necessidade de haver um maior investimento em pesquisa reportando-se a avaliação da qualidade dos serviços desse setor.

Nesse contexto, esse estudo objetivou avaliar o grau de conhecimento da equipe de enfermagem que atua na Central de Material Esterilizado, com ênfase no processo de esterilização e desinfecção dos artigos médico-hospitalares, investigando a correlação entre esse processo e a infecção do sítio cirúrgico (ISC), buscando também identificar as principais dificuldades vivenciadas no setor correlacionadas com a ocorrência de capacitação profissional.

\section{MÉTODOS}

Trata-se de uma pesquisa de campo, com abordagem quali-quantitativa e do tipo exploratória, desenvolvida na CME de um hospital público no interior da Amazônia, Santarém - PA, este oferece assistência de baixa e média complexidade para a população de Santarém-PA e toda a região Oeste.

A partir da aprovação no Comitê de Ética e Pesquisa da Universidade do Estado do Pará (UEPA), sob o parecer no 2.649.925, CAAE: 86042218.5.0000.5168. Iniciou-se o estudo entrevistando 22 profissionais da equipe de enfermagem, sendo enfermeiros, auxiliares e técnicos.

Foram inclusos os colaboradores que se disponibilizaram a participar voluntariamente mediante a assinatura do Termo de Consentimento Livre e Esclarecido (TCLE) com a aplicação de um questionário semiestruturado contendo 18 questões. Os trabalhadores de enfermagem que se encontravam em período de férias ou licença trabalhista foram excluídos. Não houve recusa de nenhum dos profissionais abordados.

A análise do material ocorreu através da técnica de "Análise Temática de Conteúdo" (Bardin, 1977), sendo um recurso que se adequa à proposta da pesquisa, formulando temas a partir dos relatos mais relevantes dos entrevistados. Posteriormente, os dados quantitativos foram transcritos ao Microsoft Office Exceß ${ }^{\circledR} 2016$, para melhor organização e auxílio analítico, além de proporcionar o armazenamento do material coletado.

\section{RESULTADOS E DISCUSSÃO}

Os colaboradores da CME foram investigados em relação ao gênero que mostrou um quantitativo em que 4 deles (18\%) eram do sexo masculino e 18 (82\%) feminino. Observou-se o predomínio do sexo feminino e implica-se tal fato ao contexto histórico da enfermagem no alcance dessa área como ciência, tendo como representantes nessa conquista mulheres que buscavam reconhecimento profissional (MACHADO RR e GELBCKE FL, 2009). A tabela 1 apontou que a idade predominante foi acima de 35 anos (50\%), seguido de 30-35 anos (23\%), 24-29 anos (18\%) e, por fim, entre 18-23 (9\%).

Tabela 1 - Idade dos profissionais de enfermagem participantes.

\begin{tabular}{ccc}
\hline Idade & N & $\%$ \\
\hline $18-23$ anos & 2 & $9 \%$ \\
$24-29$ anos & 4 & $18 \%$ \\
$30-35$ anos & 5 & $23 \%$ \\
Acima de 35 anos & 11 & $50 \%$
\end{tabular}

Fonte: Dados da pesquisa, 2018. 
Costa CCP, Souza NVDO e Pires AS (2016) e Neis MEB et al. (2011) afirmam que a maioria dos colaboradores da CME possuem idade mais avançada, tornando-os menos motivados e com maior tempo de desgaste no trabalho, concordando com a presente pesquisa. Silva PSC, Santos MV e Costa CRM (2013), Costa CCP, Souza NVDO e Pires AS (2016), Carvalho PA et al. (2016), Bugs TV et al. (2017) destacam que a idade é um fator importante, uma vez que, o vigor profissional influencia na qualidade dos serviços.

Em relação à categoria profissional, a amostra foi composta por 4 enfermeiros e 18 técnicos de enfermagem, correspondendo respectivamente a 18\% e 82\%. Neis MEB e Gelbcke FL (2013) e Costa CCP, Souza NVDO e Pires AS (2016) expõem que grande parte dos serviços do setor são desempenhados pelos técnicos e auxiliares de enfermagem, logo, há necessidade de haver maior número desses profissionais em relação aos enfermeiros.

Após a análise das atividades que os técnicos em enfermagem realizam na CME (Tabela 2), considerando as etapas do processamento de materiais, a pesquisa mostrou que $8(45 \%)$ atuam na limpeza, desinfecção, esterilização e empacotamento, $3(17 \%)$ apenas no empacotamento, $3(17 \%)$ somente na limpeza e empacotamento, $1(5 \%)$ limpeza e desinfecção, 1 (5\%) esterilização e 2 (11\%) não responderam este item do questionário.

Tabela 2 - Funções dos técnicos em Enfermagem da CME.

\begin{tabular}{ccc}
\hline Funções & N & $\%$ \\
\hline Empacotamento/armazenamento & 3 & $17 \%$ \\
Esterilização & 1 & $5,5 \%$ \\
Limpeza/desinfecção & 1 & $5,5 \%$ \\
Limpeza/desinfecção e & 3 & $17 \%$ \\
empacotamento/armazenamento & & \\
Todas as funções & 8 & $44 \%$ \\
Não responderam & 2 & $11 \%$
\end{tabular}

Fonte: Dados da pesquisa, 2018.

Observa-se que a maior parcela dos funcionários desempenham todas as funções dentro da CME, desde o recebimento até a distribuição, por ser do tipo centralizada, achado esse que concorda com um estudo de Silva PSC, Santos MV e Costa CRM (2013), desenvolvido em uma Central, onde descreve que não há atividade específica para os funcionários, e que a divisão de tarefas é realizada perante as demandas do setor (SILVA PSC; SANTOS MV; COSTA CRM, 2013).

Considerando o tempo de serviço dos trabalhadores de enfermagem (Tabela 3), 9 (41\%) possuem de 3 a 6 anos de serviço no setor, 8 (36\%) menos de 3 anos, e 5 (23\%) trabalham na CME por mais de 6 anos.

Tabela 3 - Tempo de serviço na central de materiais e esterilização dos participantes da pesquisa.

\begin{tabular}{cll}
\hline Tempo de serviço na CME & N & $\%$ \\
\hline Menos de 3 anos & 8 & $36 \%$ \\
De 3 a 6 anos & 9 & $41 \%$ \\
Acima de 6 anos & 5 & $23 \%$ \\
\hline
\end{tabular}

Fonte: Dados da pesquisa, 2018. 
O tempo de atuação do profissional em um setor específico possibilita que o mesmo tenha maior domínio da organização e processo de trabalho, além da experiência que torna as atividades mais eficientes (COSTA CCP; SOUZA NVDO; PIRES AS, 2016).

Nos aspectos relacionados à oferta de treinamentos sobre atualizações do processo de descontaminação/esterilização e manuseio de equipamentos pela Instituição, 10 (45\%) dos participantes afirmaram que a instituição fornece educação continuada, sendo que 1 respondeu que ocorre mensalmente, 4 alegaram ser anualmente, e 5 semestralmente, havendo divergências nas informações. No entanto, 12 $(55 \%)$ dos pesquisados negaram receber treinamentos pelo hospital.

Nesse âmbito, Bugs TV et al. (2017), abordam em sua pesquisa que por se tratar de um setor de alta complexidade, as atualizações são imprescindíveis, pois são através delas que os profissionais aprendem a manusear as novas tecnologias acerca do processamento de materiais. Quanto à qualificação individual através de meios externos (minicursos, simpósios, congressos, oficinas, etc.), a pesquisa mostrou que 16 (73\%) dos colaboradores buscam capacitações para aprimorar os seus conhecimentos e $6(27 \%)$ não procuram outras oportunidades para aperfeiçoar seus saberes práticos, além daqueles fornecidos pelo hospital.

Sabe-se que o mundo contemporâneo e globalizado de hoje mostra-se cada vez mais exigente e seletivo, consequentemente com a chegada de novas tecnologias tornou-se essencial que os profissionais busquem por uma requalificação, pois a renovação constante dos conhecimentos garante o aperfeiçoamento das habilidades básicas para o desempenho de suas funções e maior valorização dos mesmos (VIDIGAL CBR e VIDIGAL VG, 2012).

Quando questionados sobre o conceito correto do processo de desinfecção e esterilização, 21 (95\%) entende que esse processamento envolve a destruição de todos os tipos de microrganismos inclusive os esporulados, e 1 (5\%) define que apenas remove as sujidades dos artigos médico-hospitalares. Garcia RA, Oliveira VE e Beltrameli M (2014), conceituam a esterilização como ação que destrói todas as formas microscópicas de vida, tornando o material livre de agentes de infecções. É perceptível que o resultado foi quase unânime entre os participantes demonstrando que eles detêm conhecimento limitado.

Em relação ao conceito de artigos críticos e não-críticos (Gráfico 1), 14 (64\%) referem serem os que entram em contato com tecidos estéreis, ou com o sistema vascular e penetram em órgãos e tecidos, e os não-críticos são os que entram em contato apenas com a pele íntegra e superfícies, $6(27 \%)$ afirmam ser artigo crítico "aqueles que não oferecem grande risco de infecção", enquanto que os não-críticos estão envolvidos com alto risco de causar infecções, e a minoria, 2 (9\%) considera que os críticos são "os que entram em contato apenas com a pele íntegra", e os não-críticos são os envolvidos em procedimentos que penetram a pele e órgãos.

Gráfico 1 - Conhecimento sobre conceito de artigos críticos e não-críticos.

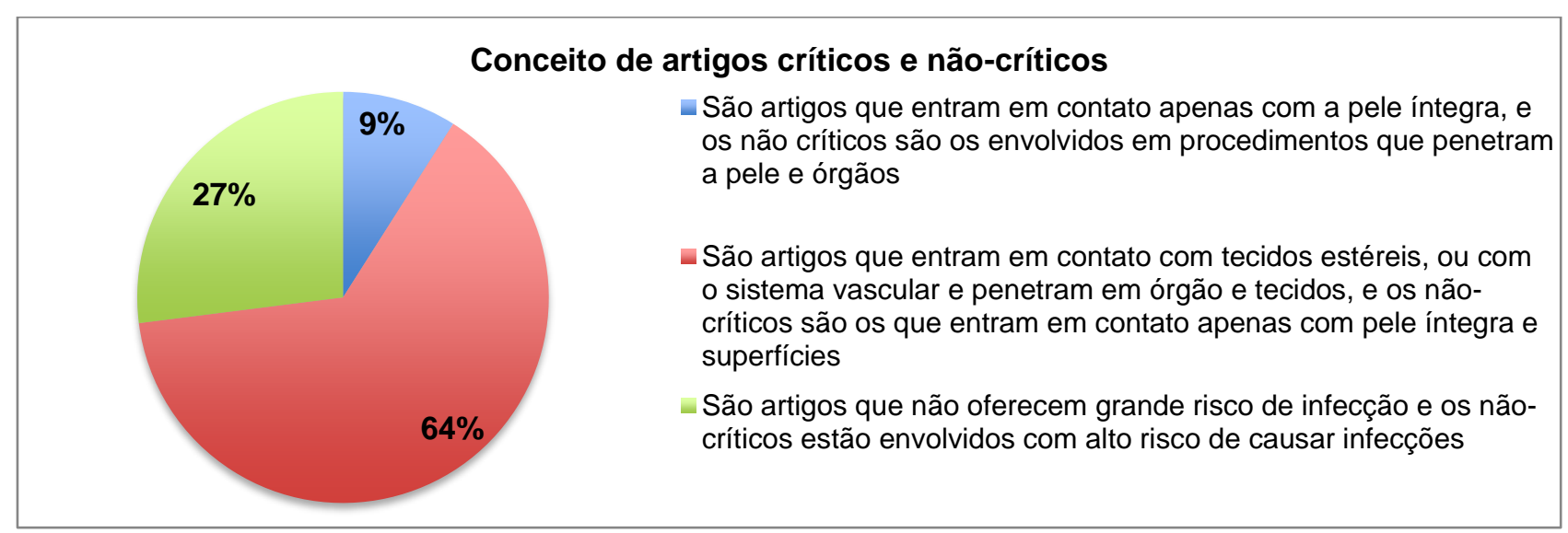

Fonte: Dados da pesquisa, 2018. 
Neste sentido, a maior parte dos funcionários sabe diferenciar um artigo crítico do não-crítico, sendo os críticos aqueles que devem ser submetidos ao processo de esterilização, e os não-críticos os que somente necessitam de desinfecção de médio ou baixo nível, quando reutilizados em pacientes, como dispõe a Resolução RDC n 15 de 15 de Março de 2012 (BRASIL, 2012).

Buscou-se avaliar também o conhecimento que eles detinham sobre o teste de Bowie e Dick (Gráfico 2) sabendo que essa ferramenta faz parte da rotina dessa CME. Evidenciou-se que $18(82 \%)$ participantes descreveram ser um teste que verifica a eficácia da esterilização, $3(14 \%)$ referiram que o teste é usado para monitorar o sistema de pré-vácuo em autoclaves a vapor com bomba de vácuo, que identifica a presença de ar no interior dos pacotes, e apenas 1 (4\%), afirmou ser um teste utilizado para testar a eficácia do processo de desinfecção de artigos.

Gráfico 2 - Conhecimento da equipe de enfermagem da CME sobre o teste de Bowie e Dick.

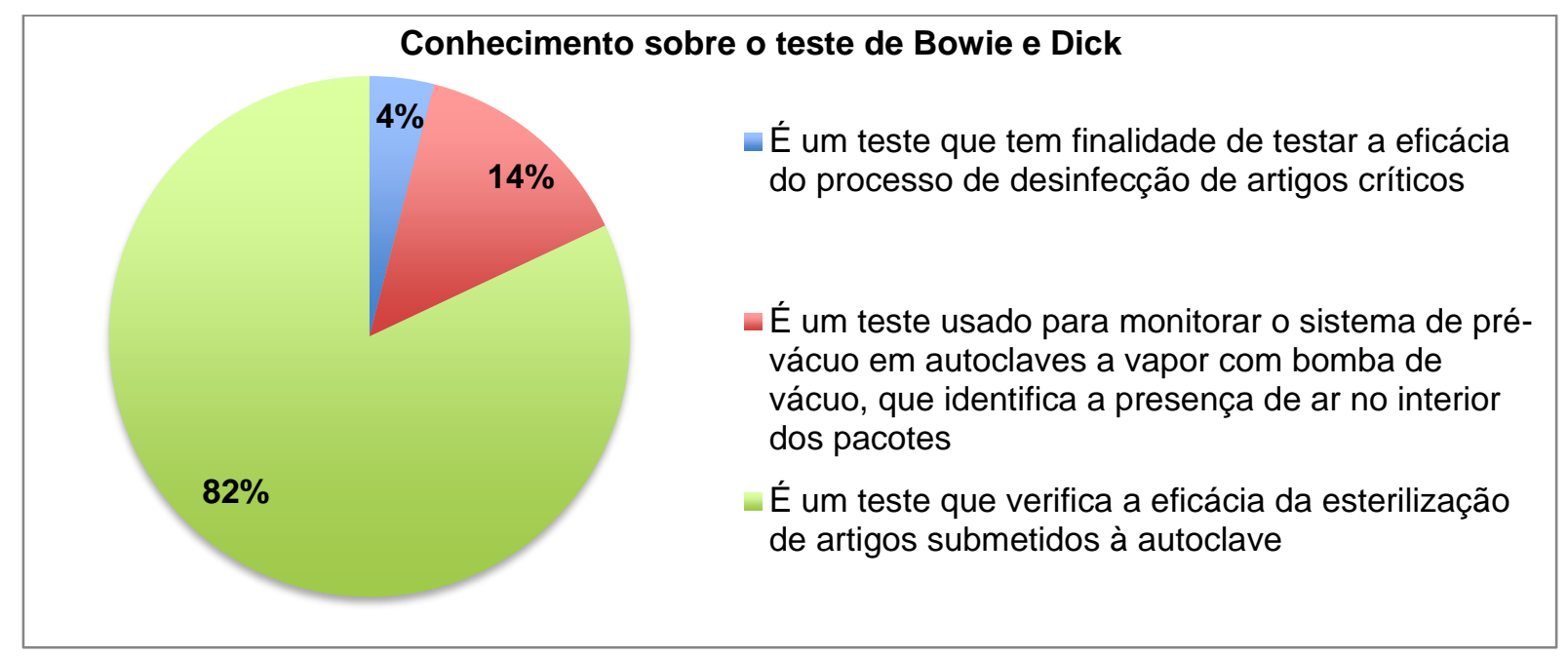

Fonte: Dados da pesquisa, 2018.

Segundo Tipple AFV et al. (2011) o teste de Bowie-Dick é usualmente escolhido para testar o reprocessamento de artigos médicos hospitalares, por apresentar uma relação custo-benefício positiva e ser de fácil manuseio. Esse indicador tem como finalidade detectar presença de ar residual que ocorre devido falhas na bomba de vácuo da autoclave, quando positiva o equipamento não deve ser utilizado, pois a presença de ar é uma barreira para a penetração do vapor.

A partir da análise das respostas abertas formou-se 4 categorias: a visão da equipe de enfermagem acerca da atuação do enfermeiro; qualidade e a importância do processamento de materiais e sua contribuição na redução das infecções hospitalares; dificuldades enfrentadas pela equipe de enfermagem na rotina da CME e a utilização dos equipamentos de proteção individual entre os trabalhadores desse setor.

\section{A visão da equipe de enfermagem acerca da atuação do enfermeiro}

O processo de trabalho do enfermeiro na CME é diversificado envolvendo funções administrativas e de coordenação. Para isso, necessita-se de conhecimento em relação ao processamento de materiais, manuseio correto das tecnologias envolvidas, liderança de equipe e gerenciamento. A partir dos relatos dos pesquisados observou-se que o trabalho do enfermeiro é visto de forma fragmentada diante das respostas incompletas:

"Organizar, orientar, treinar sua respectiva equipe, além de ser alguém que deva entender tanto do processo de desinfecção quanto do de esterilização, assim como materiais utilizados para esses fins" (Halstead).

"Observar e verificar as técnicas empregadas no CME, o modo que o material está sendo armazenado e o controle sobre a distribuição de materiais" (Kelly). 
A visibilidade do enfermeiro pode estar associada à percepção da equipe de enfermagem sobre as competências e responsabilidades desse profissional para com o setor (SANCHEZ ML et al., 2018). Nota-se que esse conhecimento escasso é um obstáculo para o entendimento desse colaborador com função também de nortear as atividades de rotinas do CME.

\section{Qualidade e a importância do processamento de materiais e sua contribuição na redução das infecções hospitalares}

Nessa categoria, o enfoque principal direciona-se ao controle das infecções hospitalares através da qualidade do processamento de artigos. Dentre as respostas, observou-se que a maioria compreende as ações que devem ser realizadas para a redução dessas infecções:

"Controlar os indicadores químicos, físicos, biológicos, principalmente o biológico que é o padrão ouro, para se saber se está tendo uma boa esterilização. Além de controlar os indicadores, anotar e protocolar e arquivar" (Mixter).

"Inserção de controle biológico, controle das etapas realizadas, controle de materiais e instrumentais, redução das possibilidades do erro humano, aumento da eficiência operacional, relatório produtivo" (Finochietto).

Ouriques CM e Machado MÉ (2013) descreve que para a validação da eficácia da esterilização devem ser realizados a supervisão e o controle dos indicadores químicos, físicos e biológicos, que são capazes de perceber falhas nos equipamentos e amenizar os riscos associados ao funcionamento inadequado dos aparelhos. A realização deste controle e a garantia da sequência correta do processamento de materiais influenciam diretamente na incidência de infecções hospitalares, trazendo maiores custos para o hospital, aumentando a permanência dos pacientes assistidos e demonstrando a qualificação da assistência prestada (PEREIRA GM, 2011).

Os sujeitos do estudo, quando questionados sobre as etapas sequenciais de descontaminação e esterilização do material cirúrgico, evidenciaram domínio quanto ao assunto, como pode ser visto nos depoimentos:

\footnotetext{
"Para esterilização: a separação, lavagem, secagem, preparo, empacotamento e processo de esterilização mecânico. Para desinfecção: lavagem e desinfecção (solução ácido, enzimático) e preparação, ambos identificados e validados" (Maynihan).

"Lavagem, secagem, empacotamento, identificação, esterilização e armazenamento" (Duval).
}

É imprescindível que os trabalhadores conheçam o sequenciamento correto do processo dos materiais, pois é o que determina se o material está estéril para ser usado e isento de contaminantes. No entanto, eles reconhecerem a importância do setor para a qualidade dos serviços do hospital também é um fator relevante para despertar o interesse em realizar as etapas do processamento corretamente. Todos os pesquisados apontaram a CME como extremamente importante, enfatizando que todas as Unidades dependem diretamente dos serviços desse setor:

"Pois o CME seria o coração de um hospital digo, caso fosse um corpo, pois é daqui que sai alguns materiais para demais clínicas para realização de procedimentos" (Kelly).

"É de fundamental importância para o desenvolvimento de cirurgias seguras ao grau de esterilização com a redução do índice de infecção hospitalar no sítio cirúrgico" (Maynihan).

Vasques JD e Damasio YLR (2016) abordam que a CME articula com todas as Unidades hospitalares, promovendo uma assistência indireta, e consequentemente, contribuindo nos cuidados diretos aos pacientes. Ribeiro JM, Bredt CSO e Santos RP (2015) frisam que as atividades desenvolvidas nessa central se 
apresentam de grande importância para garantir a qualidade e segurança dos serviços prestados que devem ser realizados com competência, destreza, habilidade e responsabilidade.

\section{Dificuldades enfrentadas pela equipe de enfermagem na rotina da CME}

Nesta temática, os pesquisados identificaram algumas dificuldades inerentes ao processo de esterilização. Percebe-se, nas respostas a seguir, que um dos empecilhos apontados está relacionado à falta de materiais básicos que deveriam ser ofertados pela instituição, impactando consideravelmente no fluxo das atividades:

"Falta de materiais, treinamento sobre CME, etc." (Kocher).

"Falta de químico, panos para empacotamento dos materiais para serem utilizado" (Reynald).

Nas pesquisas de Ouriques CM e Machado MÉ (2016) e Bugs TV et al. (2017), os entrevistados apontaram como principais dificuldades no processo de trabalho na CME a estrutura física do setor, pois dificulta o fluxo de materiais e comprometem a qualidade da esterilização, além disso também identificam a desvalorização dos profissionais como impasse para a excelência dos procedimentos realizados por eles.

Percebe-se, que os resultados obtidos no estudo discordam das literaturas disponíveis. No entanto, observou-se que o setor apresenta uma estrutura física inadequada e antiga impedindo o fluxo correto de insumos.

Porém, este fator foi apontado apenas por um participante da pesquisa, evidenciando que para os outros pesquisados não se configura como um obstáculo importante que interfere nos serviços.

\section{Utilização dos equipamentos de proteção individual entre os trabalhadores da CME}

Essa categoria descreve as respostas dos pesquisados em relação aos equipamentos utilizados por eles durante a higienização das salas, preparo e armazenamento dos materiais:

"Aventais, luvas, máscaras, protetor auricular, óculos de proteção" (Backhaus).

"Gorro, máscara, luvas (quando necessário), óculos de proteção e avental" (Kelly).

Segundo Ribeiro RP e Vianna LAC (2012), Lima CA et al. (2018), Stanganelli NC (2015), Espindola MCG e Fontana RT (2012), enfatizam que os EPI's que devem ser utilizados na CME são: gorro, avental impermeável, luvas grossas de borracha, óculos, máscaras e sapato fechado, propiciando redução dos riscos ocupacionais aos quais os trabalhadores estão expostos, como respingo de sangue e demais secreções corporais, acidentes com perfuro-cortantes, bem como a exposição aos agentes físicos (calor, ruídos, umidade), químicos (produtos desinfetantes) e ergonômicos devido aos esforços repetitivos.

Faltam equipamentos de proteção individual importantes para os trabalhadores do setor, entre eles, as luvas de borracha, avental individual e máscara de carvão ativado, que não foram mencionados pelos entrevistados da instituição.

É válido ressaltar que três participantes responderam que o hospital não disponibiliza os materiais especificamente para a CME.

\section{CONCLUSÃO}

O estudo identificou que os trabalhadores da equipe de enfermagem da Central de Material Esterilizado ainda detêm escasso conhecimento científico, muito embora, na operacionalização, observou-se um maior domínio. A partir da concepção de que a educação continuada é fundamental para qualificar o indivíduo para a prática assistencial direta ou indireta, é imprescindível maiores investimentos em treinamentos intrahospitalares, oportunizando recrutar todos os funcionários da Central, com enfoque na implementação de ações concretas voltadas principalmente ao processamento correto dos artigos médico-hospitalares e ao manuseio de inovações tecnológicas utilizadas para tornar o serviço mais hábil, devendo ser realizadas com periodicidade trimestral, melhorando assim a qualidade do serviço prestado. 


\section{REFERÊNCIAS}

1. ANJOS MAM, OLIVEIRA JC. As percepções dos profissionais de enfermagem da central de material e esterilização: uma reflexão sobre a cultura organizacional. Revista ACRED, Rio de Janeiro, 2016; 6(11): 1-9.

2. ARARUNA AB, POSSO MBS. Centro de material de esterilização: parâmetros espaciais e Riscos físicos. Revista SOBECC, São Paulo, 2014; 19(3): 142-147.

3. BRASIL. Ministério da Saúde. Resolução № 466 de 12 de Dezembro de 2012. Conselho Nacional de Saúde. Brasília - DF, 2012.

4. BRASIL. Ministério da Saúde. Resolução RDC no 15, de 15 de março de 2012. Dispõe sobre requisitos de boas práticas para o processamento de produtos para saúde e dá outras providências.

5. BORGHETI SP, et al. A Biossegurança no centro de materiais e esterilização: Dúvidas dos profissionais. Revista SOBBEC, São Paulo, 2016; 21(1): 3-12.

6. BUGS TV, et al. Perfil da equipe de enfermagem e percepções do trabalho realizado em uma central de materiais. Revista Mineira de Enfermagem, Belo Horizonte-Minas Gerais, 2017; 21.

7. CARVALHO PA, et al. Cultura de segurança no centro cirúrgico de um hospital público na percepção dos profissionais de saúde. Rev. Latino-AM. Enfermagem, Ribeirão Preto- São Paulo, 2016; 23 (6).

8. COSTA CCP, et al. Perfil dos trabalhadores de uma central de material e esterilização: uma análise das características sócio profissionais. Revista de pesquisa cuidado é fundamental online, Rio de Janeiro, $2016 ; 08$ (1): 3633-3645.

9. ESPINDOLA MCG, FONTANA RT. Riscos ocupacionais e mecanismos de autocuidado do trabalhador de um centro de material e esterilização. Rev. Gaúcha Enferm, Porto Alegre, 2012; 33 (1): 116-23.

10. FUSCO SFB, SPIRI WC. Análise dos indicadores de qualidade de centros de material e esterilização de Hospitais públicos acreditados. Florianópolis, 2014.

11. GARCIA RA, et al. Normas e rotinas para o processamento de materiais de Enfermagem/médico/odontológico. Campinas, 2014.

12. GIL RF, et al. Atividades do enfermeiro de centro de material e esterilização em instituições hospitalares, Florianópolis- Santa Catarina, 2013.

13. HOYASHI CMT, et al. A Central de material e esterilização na formação do Enfermeiro: proposta de um Manual de Práticas. Revista Práxis, Volta Redonda, 2015; Ano VII (14).

14. JACOBY AM, et al. Desinfecção e Esterilização em Serviços Ambulatoriais de Saúde do Trabalhador. Rev. Cogitare Enferm, Santa Catarina, 2016; 21 (1): 1-10.

15. LIMA CA, et al. Avaliação observacional da central de material e esterilização de um hospital universitário. Revista Eletrônica Gestão \& Saúde, 2014; 05 (03): 2010-19.

16. MACHADO RR, GELBCKE FL. Que brumas impedem a visibilização do centro de material e esterilização? Revista Texto Contexto Enferm, Florianópolis, 2009; 18 (2): 347-54.

17. NEIS MEB, et al. Centro de material e esterilização: dimensionamento de Pessoal - estudo sobre a variável tempo efetivo de trabalho. 2011.

18. NEIS MEB, GELBCKE FL. Carga de trabalho em centro de material e esterilização: subsídios para dimensionar pessoal de enfermagem. Revista Eletrônica de Enfermagem, Goiás, 2013; 15 (1): 15-24.

19. OURIQUES CM, MACHADO MÉ. Enfermagem no processo de esterilização de materiais, Florianópolis, 2013.

20. PEREIRA GM. A problemática das infecções hospitalares e o papel da Central de Material Esterilizado vivenciado em um hospital de reabilitação. Monografia (Especialização em microbiologia) - Instituto de Ciências Biológicas, Universidade Federal de Minas Gerais, Belo Horizonte, 2011; $91 \mathrm{f}$.

21. RIBEIRO JM, et al. Central de materiais esterilizados e controle de infecção hospitalar: uma revisão narrativa. Revista Varia Scientia- Ciências da Saúde, Paraná, 2015; 1(2).

22. RIBEIRO RP, VIANNA, LAC. Uso dos equipamentos de proteção individual entre trabalhadores das centrais de material e esterilização. Revista Cienc Cuid Saúde, Ribeirão Preto- São Paulo, 2012; 11: 199-203.

23. ROCHA JMN. (Ed). Central de Material Esterilizado (CME) do Ambulatório escola Dr. Valter Evaristo Amorim. Tocantins. 2015.

24. SANCHEZ ML, et al. Estratégias que contribuem para a visibilidade do trabalho do enfermeiro na central de material e esterilização. Rev. Texto Contexto Enferm, Rio Grande do Sul, 2018; 27(1).

25. SILVA AC, AGUIAR BGC. O enfermeiro na Central de Material e Esterilização: uma visão das unidades consumidoras. Revista Enfermagem UERJ, Rio de Janeiro, 2008; 16(3): 377-81.

26. SILVA PSC, et al. Atuação da enfermagem na central de material e esterilização em um hospital de Teresina. Revista Interdisciplinar, Teresina- Piauí, 2013; 6 (3): 45-51.

27. STANGANELLI NC, et al. A utilização de equipamentos de proteção individual entre trabalhadores de enfermagem de um hospital público. Rev. Cogitare Enferm. Paraná, 2015; 20 (2): 345-5.

28. TIPPLE AFV, et al. O monitoramento de processos físicos de esterilização em hospitais do interior do estado de Goiás. Rev Esc Enferm USP, São Paulo, 2011; 45 (3): 751-7.

29. VASQUES JD, DAMASIO YLR. A importância do Enfermeiro no processo de gerenciamento na Central de Material e Esterilização Hospitalar, Recife, 2016.

30. VIDIGAL CBR, VIDIGAL VG. Investimento na qualificação profissional: uma abordagem econômica sobre sua importância. Acta Scientiarum. Human and Social Sciences, Maringá-Paraná, 2012; 34 (1): 41-48. 